\title{
Developing an integrated optics laboratory at minimum cost
}

\author{
Jerome Wagner
}

Jerome F. Wagner, "Developing an integrated optics laboratory at minimum cost," Proc. SPIE 2525, 1995 International Conference on Education in Optics, (13 October 1995); doi: 10.1117/12.224023

SPIE Event: SPIE's 1995 International Symposium on Optical Science, SPIE. Engineering, and Instrumentation, 1995, San Diego, CA, United States 


\author{
Developing an integrated optics laboratory at minimum cost \\ Jerome F. Wagner \\ Rose-Hulman Institute of Technology \\ Department of Physics and Applied Optics \\ Terre Haute, Indiana 47803
}

\begin{abstract}
A little over ten years ago, the physics department at Rose-Hulman Institute of Technology introduced an applied optics program at the graduate level. Included among the five core courses required for the Master's degree, was a four credit hour course in integrated optics which consisted of both lecture and laboratory. Such a course offering required not only a working knowledge of the lecture material but also the acquisition of appropriate equipment and material to develop a laboratory for demonstrating principles presented in class. Suitable experiments would consist of the fabrication of and the coupling into such elements as planar and channel waveguides. Other experiments would involve the study of phase modulation and amplitude modulation in integrated optical devices. Expertise for offering the course was obtained through a sabbatical taken in the electrical engineering department at the University of Cincinnati in 1983. An important feature available at the university was its microelectronics fabrication laboratory. This experience provided the necessary practical knowledge required to develop an integrated optics course with a laboratory component. Because of the expense of much of the equipment required to fabricate the devices, alternative approaches had to be taken. Some of these approaches included in-house fabrication of large scale equipment, donations of older equipment and integrated optical devices from sister institutions, government educational programs, and corporate funding programs. Although, a laboratory, as originally envisioned, has not yet been fully completed, what is available at present fulfills some of the intended goals.
\end{abstract}

Keywords: integrated optics, waveguides, thin films, optics laboratory

\title{
1. INTRODUCTION
}


In the early 1980 's, the physics department at Rose-Hulman Institute of technology developed a master's degree program in the area of applied optics. The intention was to provide a terminal degree for those students who required additional knowledge beyond the baccalaureate but not as extensive as that required at the doctorate level. At about the same time a Center for Applied Optics Studies (CAOS) was created. A proposal was submitted to and approved by the Indiana Business Modernization \& Technology Corporation ${ }^{1}$ to financially support the Center. One of the purposes of the Center was that it would act as an interface between the College and industry within Indiana. Problems suggested by industry were to be used to satisy the thesis requirement of the degree program. In addition, an occasional project was accepted from sources not located in Indiana and thesis projects were also provided internally by faculty participating in the degree program.

The program consisted of five core courses most of which contained a laboratory component. A research thesis provided the additional credits needed for satisfying the degree requirments. One of the core courses involved the technology associated with the field of integrated optics ${ }^{2}$. The lecture component of the course consisted of three periods of instruction per week. The lecture component was supplemented with a laboratory which consisted of one three-period meeting per week and was held eight of the ten weeks of the quarter. The purpose of this presentation is to describe the evolution of the course and, in particular, how equipment was obtained to support the laboratory component.

\section{BACKGROUND PREPARATION}

In preparation for offering a course in integrated optics, the author requested a sabbatical leave in 1983 to spend a year in the Electrical Engineering Department at the University of Cincinnati ${ }^{3}$. Knowledge of the appropriate material was acquired by taking several theory courses in the area of microelectonics. Device fabrication knowledge was acquired in separate laboratory courses. This experience proved to be very helpful in the development of the integrated optics course. However, lack of resources made it very difficult to provide a laboratory environment at Rose-Hulman such as the one at the sabbatical institution. In June of 1991, the author attended an NSF sponsored Microfabrication Laboratory Workshop held at San Jose State University ${ }^{4}$. The theme of the workshop was that Microfabrication experiments can be simple and inexpensive. This workshp was helpful in that it suggested alternatives to the standard fabrication methods which required expensive equipment. 


\section{COURSE MATERIAL}

Over several offerings of the course, the course material was selected from texts authored by Hunsperger ${ }^{5}$, Lee ${ }^{6}$, and Yariv-Yeh ${ }^{7}$. In the latter reference, the course was developed around a chapter of significant length and supplemented with material from other texts. The lecture component included the following topics:

1. Basic Electromagnetic Theory,

2. General Properties of Dielectric Waveguides,

3. Slab Dielectric Waveguides,

4. Practical Waveguiding Geometries,

5. Waveguide Fabrication,

6. Fabrication Lithography,

7. The Prism and Grating Couplers,

8. Dielectric Perturbation and Mode Coupling,

9. Electro-optic Modulation and Mode Coupling.

One of the classroom activities consisted of an analysis of planar waveguides. Two possibilities were considered. One structure was composed of three insulating regions (cover, waveguide, and substrate) while the other structure included one semi-conducting region. This modification introduced a complex index of refraction resulting in complex mode indicies and also permitted the student to study waveguide losses. The equation describing the discrete electromagnetic modes in the waveguide is given $\mathrm{by}^{7}$

$$
\tan h t=\frac{p+q}{h\left(1-p q / h^{2}\right)}
$$

where $t$ is the waveguide thickness, and $q, h$, and $p$ are the transverse propagation constants in the the cover, waveguide and substrate, respectively. This equation can be used for either $T E$ or $T M$ modes, however, the values of the transverse propagation constants will differ. A more useful form of Eq. (1) for $T E$ modes is

$$
f\left(n_{e m}\right)=m \pi-2 \pi\left(\frac{t}{\lambda}\right) \sqrt{n_{2}^{2}-n_{e m}^{2}}+\tan ^{-1}\left(\frac{n_{e m}^{2}-n_{1}^{2}}{n_{2}^{2}-n_{e m}^{2}}\right)^{1 / 2}+\tan ^{-1}\left(\frac{n_{e m}^{2}-n_{3}^{2}}{n_{2}^{2}-n_{e m}^{2}}\right)^{1 / 2}
$$


where $m=0,1,2 \ldots$ is the mode number, $\lambda$ is the vacuum wavelength, $n_{e m}$ is the $m$ th effective waveguide index, and $n_{1}, n_{2}$, and $n_{3}$ are the indicies of the cover, waveguide, and substrate, respectively, for that wavelength. The mode index values may be obtained by determining when

$$
f\left(n_{e m}\right)=0
$$

The students were required to write computer code to determine the solutions to the guidance equation, Eq. (2), for three cases. In the all-dielectric structure, the bisection ${ }^{8}$ method was apropriate. In the structure containing a semiconducting substrate (silicon), the Newton-Raphson method ${ }^{9}$ was adequate to determine the complex effective mode indicies. A perturbation technique ${ }^{7}$ was helpful in determining initial values for the iteration process. The third case considered the possibility of a gradient index waveguide in which case the waveguide-substrate phase term in Eq. (2) was replaced by an integral. Application of Simpson's rule ${ }^{10}$ proved satisfactory for evaluating the integral. Alternatively, solutions to the guidance condition were also obtained using MAPLE or MATHEMATICA.

\section{EXPERIMENTS}

\subsection{Ellipsometry}

The first experiment performed by the students consisted of measuring the optical properties of thin films and substrates. The measurements of the film thickness and film index were made with an ellipsometer ${ }^{11}$. The test sample was the calibration disk which accompanied the ellipsometer. The sample was a 4" silicon wafer with a thermally grown oxide layer having a thickness of $760 \AA$ and index 1.470. After reviewing the theory for the general equation of ellipsometry, the students were able to determine values for the polarization parameters $\Psi$ and $\Delta$, which in turn (computer program provided) led to the film's thickness and index. To familiarize the student with the operation of an ellipsometer, the data was taken in manual mode. The automatic mode feature was then used and a comparison of the results from the two methods was made. Each student was required to write a short program to verify the validity of the ellipsometry equation for planar structures. The support for this equipment was provided by IBMTC ${ }^{1}$.

\subsection{Planar waveguide structures}

Several methods for obtaining planar waveguides will be described. 


\subsubsection{Spun waveguides}

The laboratory experiments were designed so that the students were able to make simple planar waveguide structures, measure the optical properties of the components, and couple electromagnetic energy into the waveguide. Once coupling was achieved, the effective mode indicies could be determined and compared to those calculated from the theory.

Waveguides can be fabricated in several ways. The original intention was to evaporate a thin layer (about $1 \mu \mathrm{m}$ ) of $\mathrm{Al}_{2} \mathrm{O}_{3}$ onto a glass substrate using an electron-beam evaporator donated by General Motors ${ }^{12}$ for educational purposes. Although the evaporator was designed for the metalization process in device fabrication, one could, in principle, evaporate other materials. Unfortunately, $\mathrm{Al}_{2} \mathrm{O}_{3}$ evaporates at a much higher temperature than does $A l$. The higher temperature caused the copper boat containing the waveguide material to evaporate and the resulting waveguide film was contaminated with copper. In addition, due to the age of the evaporator, it exhibited a high failure rate and the maintenance cost was prohibitive. It was decided to try a simpler approach. A step-index waveguide can be produced by spinning an appropriate material in liquid form onto a substrate. In our case, the liquid was a positive photoresist which, when spun onto the substrate at $5000 \mathrm{rpm}$, produced a film about $1 \mu \mathrm{m}$ thick. The photoresist was then hardened by baking the structure in a vacuum oven. The spinner and the photoresist were donated by a government agency ${ }^{13}$ and the oven was donated by an educational institution ${ }^{3}$.

\subsubsection{Thermally grown waveguides}

Another method of waveguide fabrication is to thermally grow an oxide layer of silicon. After several days of growth which produces a thin waveguide film of about $1 \mu \mathrm{m}$, the furnace temperature is increased and a thick oxide substrate layer of about $10 \mu \mathrm{m}$ is grown. However, since the index difference in the oxide layers is small, mode confinement is weak.

\subsubsection{Ion - migration waveguides}

Finally, a graded-index dielectric waveguide can be fabricated using an ion-migration process $^{6}$ consisting of soda-lime glass, which contains approximately $13 \%$ of $\mathrm{Na}_{2} \mathrm{O}$ by 
weight, immersed in a molten bath of salt $\mathrm{AgNO}_{3}$. Typical melt temperatures for the silver salt range between 200 and $350^{\circ} \mathrm{C}$ with applied electric field strengths in the 0 $5 \times 10^{5}-V / m$ range. The presence of diffused silver near the glass substrate produces an increase of refractive index of about 0.1 . The diffusion time for this process is very large. A guide capable of supporting 13 modes requires diffusion for 6 hours at $250^{\circ} \mathrm{C}$.

\subsection{Channel waveguide structures}

The fabrication of channel waveguides requires equipment not easily affordable. However, several guides were acquired by donation from a former Rose-Hulman student ${ }^{16}$ who recently completed his $\mathrm{Ph} . \mathrm{D}$. work at $\mathrm{CREOL}^{17}$. The imbedded waveguides were produced by ion-exchange ( $K^{+}$in melt $\leftrightarrow N a^{+}$in glass).

\subsection{Waveguide coupling}

There are several devices which are used to convert beam energy into a mode which is guided by a thin-film dielectric layer. These devices are referred to as beam couplers. The two most common longitudinal beam couplers are the prism coupler ${ }^{14,6}$ and the grating coupler ${ }^{15}$. These are suitable when coupling into planar waveguides. Channel waveguides require transverse coupling ${ }^{15,18}$ in which the laser beam is focused on an exposed portion of the waveguide.

\subsubsection{Prism coupling}

A prism coupler is essentially a glass prism having the highest index of refraction in the system (prism plus waveguide structure). The shape of the prism may be that of an almost right triangle which is flattened on the top. This allows pressure to be applied to the prism to reduce the distance between the prism base and the waveguide surface. The prism would serve as either an input or an output coupler. Normally, one prism would be used for beam input and a second prism for beam output. A prism with a trapezoidal shape would act as both an input and output coupler.

The coupling of energy into a waveguide using a prism involves the principle of frustrated total internal reflection. The beam incident on the prism surface refracts and strikes the base of the prism. The angle of incidence is adjusted so that total internal reflection occurs at the prism base. If the prism base is close enough the the waveguide 
surface, the evanescent field can extend into the waveguide. With an additional adjustment in the angle of incidence, mode coupling will occur if the phase of the evanescent field can be matched to that of a mode. Both $T E$ and $T M$ modes are accessible.

In this part of the experiment, the student would first determine the index of refraction of the prism with the ellipsometer and would then mount the prism and the waveguide structure vertically on a goniometer. The direction of the laser beam would be fixed while the angle of incidence with the prism face was adjusted by positioning the goniometer. In a multimode waveguide, each mode could be coupled into individually. However, due to scattering, all modes were observable simultaneously. Since all of the optical parameters of the waveguide structure had been previously measured, it was left to the student to determine the mode indicies. These were then compared to the theoretical values obtained by solving the guidance equation. The equation relating the mode index to the angle of incidence of the laser beam is

$$
n_{e m}=n_{4} \sin \left[\sin ^{-1}\left(\frac{n_{1}}{n_{4}} \sin \theta_{1 m}\right)+\epsilon\right]
$$

where, $\epsilon$ is the prism base angle, $\theta_{1 m}$ is the laser beam angle of incidence, and $n_{1}$ and $n_{4}$ are the indicies of the surrounding medium and the prism, respectively.

Another experiment might involve the determination of the index of refraction and thickness of the waveguide. This would require a multimode waveguide but would still only involve the careful measurement of the beam angle of incidence for each mode.

\subsubsection{Grating coupling}

Under appropriate conditions, the grating coupler functions to produce a phase matching between a particular waveguide mode and an unguided optical beam which is incident at an oblique angle to the surface of the waveguide. Because of the periodic nature of the grating, waveguide modes in the region beneath the grating are perturbed in such a way that each mode is comprised of a set of spatial harmonics with tangential propagation constants given $\mathrm{by}^{5}$ (phase matching condition)

$$
\beta_{\nu}=\beta_{o}+\frac{2 \pi \nu}{\Lambda}
$$

where $\nu=0, \pm 1, \pm 2, \ldots$, and where $\Lambda$ is the grating periodicity. The fundamental factor $\beta_{o}$ is approximately equal to the $\beta_{m}$ of the particular mode in the waveguide region not covered by the grating. Because of the negative values of $\nu$, Eq. (5) can be satisfied so that

$$
\beta_{\nu}=k n_{1} \sin \theta_{m}
$$


even though $\beta_{m}>k n_{1}$. In Eq. (6), $n_{1}$ is the cover index, $k$ is the vacuum propagation constant, and $\theta_{m}$ is the beam angle of incidence.

A grating can be fabricated on top of a planar waveguide holographically ${ }^{19}$, producing a grating period given by

$$
\Lambda=\frac{\lambda}{2 n \sin \theta}
$$

where $\lambda$ is the vacuum laser wavelength, $n$ is the index of refraction in the medium in which the two waves interfere, and $\theta$, the beam angle of incidence in the same medium. The arrangement for fabricating the grating consists of a waveguide-substrate structure with a photoresist layer spun onto the waveguide. This structure is mounted at right angles to a mirror ${ }^{20}$. The photoresist layer is exposed by rays coming directly from the incident beam and by rays reflected from the mirror which interfere with eah other. The photoresist grating can be used as is, or, the pattern can serve as a mask for an ion milling process in which the grating is etched into the waveguide.

\subsubsection{End - fire coupling}

To couple into a channel waveguide, a X10 microscope objective is used to focus the output of a laser onto a polished endface of the waveguide. Micromanipulators are used to align the waveguide with the focused spot of light. A second microscope objective is used to couple light out of the waveguide. The emerging light can be observed on a screen and takes on the appearance of a rising sun type of diffraction pattern.

\subsection{Directional couplers}

When suitable samples can be obtained, it is anticipated that experiments will be developed involving directional and contra-directional couplers, and phase and amplitide modulation devices. Appropriate electronic and optical equipment is available in our existing laboratories to support these experiments.

\section{LITHOGRAPHY MODELING}

A small part of the course was devoted to introducing the student to the techniques required for printing small features onto substrates. A large amount of high resolution

mask technology is used in the electronics industry for the manufacture of integrated 
circuits $^{15}$. Some of this technology can be carried over to optical waveguide technology. Computer modeling programs are available which allow simulation of the lithography process. A donation of one of these programs, PROLITH $/ 2^{21}$, to the Institute was used for the development of several laboratory exercises.

\section{ACKNOWLEDGEMENTS}

The author would like to thank all those who donated equipment for this project and also thank those collegues who supported this effort.

\section{REFERENCES}

1. Indiana Corporation for Science and Technology, Grant No. P-4097 (CAOS).

2. S. E. Miller, "Integrated Optics: An Introduction," Bell Syst. Tech. J., 48, 2059 (1969).

3. Department of Electrical and Computer Engineering, College of Engineering, University of Cincinnati, Cincinnati, OH 45221-0030.

4. Center for Electronic Materials and Devices, College of Engineering, San Jose State University, San Jose, CA 95192-0080.

5. R. G. Hunsperger, Integrated Optics: Theory and Technology, 2nd ed., Springer Verlag, New York, 1983.

6. D. L. Lee, Electromagnetic Principles of Integrated Optics, Wiley, New York, 1986.

7. A. Yariv and P. Yeh, Optical Waves in Crystals, Wiley-Interscience, New York, 1984, Chapter 11.

8. W. H. Press, B. P. Flannery, A. A. Teukolsky, and W. T. Vetterling, Numerical Recipes - The Art of Scientific Computing, Cambridge University Press, New York, 1986, Chapter 9.1.

9. W. H. Press, B. P. Flannery, A. A. Teukolsky, and W. T. Vetterling, Numerical Recipes - The Art of Scientific Computing, Cambridge University Press, New York, 1986, Chapter 9.4 . 
10. W. H. Press, B. P. Flannery, A. A. Teukolsky, and W. T. Vetterling, Numerical Recipes - The Art of Scientific Computing, Cambridge University Press, New York, 1986, Chapter 4.1 .

11. Gaertner Scientific Corporation, 1201 Wrightwood Ave., Chicago, IL 60614.

12. General Motors Corp., Delco Division, Kokomo, IN.

13. National Security Agency, Central Security Service, 9800 Savage Road, Fort George G. Meade, MD, 20755-6000.

14. T. Tamir, Topics in Applied Physics: Integrated Optics, Vol. 7, Springer-Verlag, New York, Chapters 3.1.2, 5.2.4.

15. T. Tamir, Topics in Applied Physics: Integrated Optics, Vol. 7, Springer-Verlag, New York, Chapters 3.1.3.

16. Michael Sundheimer, CREOL, University of Central Florida.

17. Center for Research in Electro-optics and Lasers, University of Central Florida, Orlando, Florida 32826.

18. Richard Syms and John Cozens, Optical Guided Waves and Devices, McGraw-Hill, New York, 1992, Chapter 9.

19. Yu.I.Ostrovsky, M.M. Butnsov, G.V. Ostrovskaya: Interferometry by Holography, Springer Ser. Opt. Sci., Vol. 20 (Springer, Berlin, Heidelberg, New York, 1980).

20. M. Sundheimer, Photoresist Grating Fabrication, CREOL.

21. Finle Technologoes, P.O. Box 162712, Austin, Texas 78716. 